\title{
Salicornia ramosissima ethanolic extract on mice: a light microscopy approach on liver and kidney
}

\author{
D. Ferreira*, D. Pinto**, H. Silva*** and M.L. Pereira*
}

* Department of Biology \& Centre for Research in Ceramics and Composite Materials (CICECO), University of Aveiro, Campus de Santiago, 3810-193 Aveiro, Portugal

** Department of Chemistry \& Organic Chemistry, Natural and Agro-food Products (QOPNA) University of Aveiro, Campus de Santiago, 3810-193 Aveiro, Portugal

*** Department of Biology \& Centre for Environmental and Marine Studies (CESAM), University of Aveiro, Campus de Santiago, 3810-193 Aveiro, Portugal

Salicornia ramosissima J. Woods (Chenopodiaceae), included in the species aggregate $S$. europaea agg., is an annual succulent halophyte and one of the most salt tolerant plants, broadly distributed in the salt marshes and salt pans of Ria de Aveiro and in many others of the Iberian Peninsula [1]. Salicornia L. has been used not only as a seasoned vegetable, salad and fermented food in coastal areas of Europe and Asia [2], but also as folk medicine for disorders such as constipation, obesity and diabetes [3]. To corroborate this, the literature reports immunomodulatory, antioxidative, anti-inflammatory, anti-hyperlipidemic and antidiabetic effects [4]. Moreover, some bioactive compounds from its aerial parts were recently isolated and identified, exhibiting also antioxidant and cytotoxic activities $[2,5]$.

Factors such as the proper botanical identification, season and harvest site, extraction and purification methods, characterisation of the active constituents and the extract effects in a dose-and time-dependent manner are crucial to assess the therapeutic potential of herbal extracts [6]. The aim of this study was to investigate the possible hepatic and renal effects of an ethanolic extract of $S$. ramosissima on mice.

Aerial portions of $S$. ramosissima were collected from a salt pan in Ria de Aveiro (Portugal). The ethanolic extract $(50 \mathrm{mg} / \mathrm{kg}$ b.w.) was orally administered, during 3 weeks, to male ICR-CD1 mice, purchased from Harlan (Spain). Control group was also considered. Liver and kidney were collected and prepared for histology. Animal procedures were followed according to guidelines for ethics and animal care.

Central and portal vein congestion and severe hydropic changes of the hepatocytes were noted in the liver of exposed group, compared with controls (Figs. 1A, 1B). Renal profile of control group evidenced normal features (Figs.1C, 1E). However, significant histological alterations were found in the exposed group: cortical interstitial haemorrhages (Fig. 1D), inflammatory cell infiltration and tubular cell degeneration within medulla and corticomedullary junction (Fig. 1F).

In conclusion, this pilot study has demonstrated considerable effects in the mouse metabolism of S. ramosissima ethanolic extract, with significant hepatic and renal lesions. Further studies are needed to correlate these data with isolated active constituents for a more reliable evaluation of the potential of this species.

\section{References}

1. Silva H. et al., Ecol. Res., 22:125-134, 2007.

2. Kim J.Y. et al., Food Chem., 125:55-62, 2011.

3. Lee K.Y. et al., J. Ethnopharmacol., 103:372-378, 2006.

4. Rhee M.H. et al., J. Med. Plants Res., 3:548-555, 2009.

5. Wang Q. et al., Fitoterapia, 83:742-749, 2012.

6. Bandaranayake W.M., In: Ahmad I., Aqil F. and Owais M., eds. Modern Phytomedicine: Turning Medicinal Plants into Drugs, Weinheim: Wiley, 25-57, 2006. 

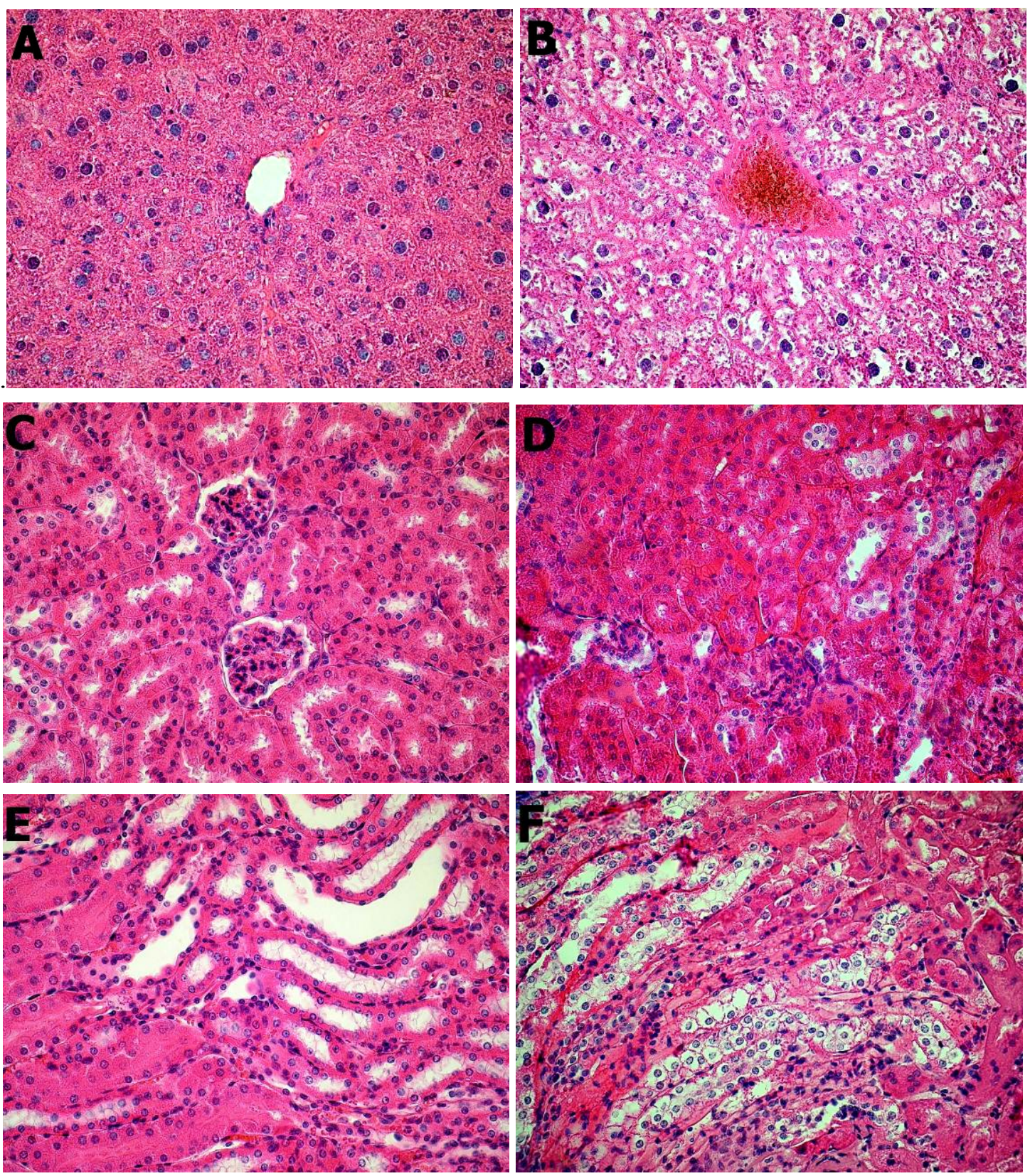

Fig 1. Light microphotographs of liver (A, B) and kidney sections (C-F) (H\&E stain, 400× original magnification): A, C and E) Control group; B, D and F) S. ramosissima ethanolic extract-treated group.

Acknowledgements

Grants were provided by CICECO, QOPNA and CESAM. 